Dialectologia 24 (2020), 23-42.

ISSN: 2013-2247

Received 22 April 2018.

Accepted 26 August 2018.

\title{
LÉXICO PATRIMONIAL MIRANDÉS: ANÁLISIS COMPARATIVO
}

\author{
Vicente Álvarez VIVES \& Melanie AzeVEdo SAMPAIO \\ Universität Paderborn * \\ alvarez@mail.upb.de / melanie.sampaio@web.de
}

Resumen

La siguiente contribución se dedica al estudio del léxico patrimonial mirandés, concretamente, al análisis de seis términos mirandeses que han sido recopilados en una obra literaria actual. A través de su traducción en portugués y en español, y tras la consulta en varios diccionarios etimológicos, se ha trazado un breve análisis histórico de cada término.

\section{Palabras clave}

mirandés, lenguas en contacto, variedades, diccionarios, regionalismos

\section{MIRANDESE LEXICON: COMPARATIVE ANALYSIS}

\section{Abstract}

The following contribution comprises the research on the patrimonial Mirandese lexicon, or, more precisely, the analysis of six Mirandese terms which have been collected from a recent literary work. A brief historical analysis of each word will be presented, based on its translation into Portuguese and Spanish and on the consultation of various etymological dictionaries.

\section{Keywords}

Mirandese language, languages in contact, varieties, dictionaries, regionalism

* Institut für Romanistik. Faculty of Arts and Humanities. University of Paderborn. Warburger Str. 100, 33098 Paderborn. Germany. 


\section{Introducción}

A propósito de las variedades fronterizas, en el marco de lenguas en contacto, Medina López (2002: 31) señala que existen «amplias zonas de toda la frontera portuguesa-española con dialectos de base leonesa como el de Ermisende (Zamora), Riodonor (Zamora-Braganza), Guadramil (Braganza), Miranda y Sendim (Portugal)». Se trata de regiones en las que la frontera lingüística no coincide con la frontera político-administrativa, sea del país, sea de la región, y en las que se emplean dos o más lenguas o variedades. Incluso, se puede llegar a crear «una nueva «modalidad de frontera» constituida por elementos de las dos lenguas» (Medina López 22002: 29).

En el extremo nordeste de Portugal, en el distrito de Braganza, se encuentran algunos dialectos o falares (hablas) no gallegoportugueses que, a pesar de su aislamiento geográfico y escaso número de hablantes, consiguieron sobrevivir desde sus orígenes en la Edad Media hasta la actualidad: el riodonorés, el guadramilés y el mirandés. El presente artículo ${ }^{1}$ se ocupa exclusivamente de la Ihéngua mirandesa, ya que ha recibido una atención especial en los últimos años por haber sido reconocida oficialmente en 1999 como lengua minoritaria de Portugal. Debido al hecho de que el mirandés es hablado en una región portuguesa que colinda con España, se observan en su sistema lingüístico abundantes coincidencias tanto con el portugués como con el español. A principios del siglo XX, José Leite de Vasconcelos (1901: 3-4) dio a conocer la existencia del mirandés como variedad lingüística, y ya anotó la relación entre las tres lenguas que entran en contacto en estos territorios: «[A]chamos que o mirandês tem $a+b$ em commum com o português, e $e+f$ em commum com o hespanhol»² (Leite de Vasconcelos 1901: 67-68):

\footnotetext{
${ }^{1}$ Basado en un trabajo escrito por Melanie Azevedo Sampaio, realizado como Trabajo de Fin de Grado (TFG - Bachelor of Education) en el Departamento de Lenguas y Literaturas Románicas de la Universidad de Paderborn en el año 2017, dirigido por Vicente Álvarez Vives y titulado: «L mirandés: El desarrollo de una variedad lingüística en la frontera hispano-portuguesa».

${ }^{2}$ La palabra hespanhol con $<\mathrm{h}>$ responde a la ortografía portuguesa de finales del siglo XIX.
} 


$$
\begin{aligned}
& a+b+c+d=\text { A é o português, } \\
& \begin{array}{c}
c+d+e+f=\text { B é o hespanhol, } \\
a+b+e+f=\text { C é o mirandês }
\end{array}
\end{aligned}
$$

Según la anterior representación esquemática, el mirandés sería una lengua solamente formada por rasgos portugueses y españoles. Sin embargo, hoy en día, un siglo después de los estudios de Leite de Vasconcelos sobre el mirandés, se encuentran no solo palabras que se asemejan a las lenguas prestigiosas, sino también varios términos o características lingüísticas que contribuyen a la individualización del idioma mirandés. Además, según Azevedo Maia (1996: 165), el hecho de que el mirandés incluso permite estructurarse en tres subvariedades diatópicas (subvariedad central, subvariedad rayana y sendinés) no solo pone de relieve su diversidad, sino también su vitalidad.

A continuación, se dará, en primer lugar, una concisa descripción del origen, contexto, geografía y de la situación actual del mirandés. En segundo lugar, se analizarán seis términos mirandeses a partir de una obra literaria, originalmente escrita en portugués por el padre José Telmo Ferraz en 1960, y que, en 2016, fue publicada en una versión bilingüe (portugués-mirandés), titulada $O$ Lodo e as Estrelas / L Lhodo i las Streilhas, traducción firmada por Fracisco Niebro. Esta versión nos permite contrastar los equivalentes del mirandés con los del idioma portugués.

\section{El mirandés: variedad fronteriza}

Como se ha comentado anteriormente, en el distrito de Braganza (Portugal), en la región de Trás-os-Montes, se encuentra la ciudad de Miranda do Douro y, tanto allí como en sus alrededores, en las freguesias ${ }^{3}$ del concejo de Vimioso y de Miranda, se habla un idioma que históricamente no forma parte del sistema gallegoportugués: I

\footnotetext{
${ }^{3}$ En Portugal, el término freguesia equivale al de parroquia en español (GDLP: 165).
} 
mirandés (Merlan 2009: 55-57). Se trata de una variedad lingüística perteneciente a la familia de las lenguas asturleonesas que es hablada por cerca de 10.000 personas. ${ }^{4}$

\subsection{Contexto y geografía}

A lo largo del tiempo, particularmente en los siglos XIX y XX, ha habido filólogos (Leite de Vasconcelos 1882; Menéndez Pidal 1906) que se han cuestionado ¿cómo es posible la existencia de una variedad, que no es la gallegoportuguesa, en territorio lusitano? Cabe destacar que la presencia de dicha variedad no es evidente a primera vista, ya que ésta se debe a motivos históricos que nos remontan a muchos siglos atrás. Sin embargo, existe la tesis histórica de Menéndez Pidal (1906: 132), según la cual, la Terra de Miranda, donde se hablan los dialectos no portugueses ya mencionados, no formaba parte del convento jurídico de Bracara Augusta en la Hispania Romana, sino del convento de Asturica Augusta. En los siglos posteriores, entre los siglos VII y XII, la iglesia de Braganza estuvo adscrita a la diócesis de Astorga en lugar de a la de Braga, lo cual intensificó el intercambio lingüístico. Solamente a partir del año 1143, cuando Portugal consiguió independizarse del Reino de León, tuvo lugar la desmembración de la diócesis astorgana por la dificultad de estar ligada a reinos distintos (Menéndez Pidal 1906: 132). Esta breve mirada histórica explica la presencia del mirandés, entendiéndolo como un enclave lingüístico asturleonés en el rincón nordeste de la República Portuguesa.

Aún hoy, casi un milenio después de que Portugal se desligara del Reino de León, el mirandés sigue viviendo, si bien en un área más pequeña, en la extremidad norte del país lusitano. Transmitido exclusivamente por el lenguaje oral de generación en generación hasta el año 1884 y siempre oponiéndose al prestigioso portugués, cabe preguntarse cómo el «astur-leonés de Portugal» (Ferreira 2010), que solamente es hablado por menos del $1 \%$ de la población portuguesa, ha conseguido mantenerse durante tanto tiempo. Hay abundantes razones que contribuyeron y todavía contribuyen a la conservación del mirandés; sin embargo, en este artículo se

\footnotetext{
${ }^{4}$ Este número aproximado también incluye los 5.000 emigrantes de las últimas décadas (Merlan 2009: 77-78).
} 
tratarán los factores esenciales. En primer lugar, la posición geográfica de la Terra de Miranda desempeña un papel importante. Por ser la región más alejada del centro de Portugal, fue siempre habitual para sus habitantes entrar en contacto con las regiones fronterizas contiguas, especialmente con la gente de Aliste y Sayago, en vez de relacionarse con los portugueses (Merlan 2009: 68-69). Otro aspecto indispensable en el mantenimiento de la variedad autóctona es el rol y el desarrollo de la economía de Miranda. Mientras que la mayor parte de Portugal pasó de una economía agraria basada en el trabajo campesino y ganadero a una economía industrial en el siglo XIX, el territorio mirandés se opuso al proceso de industrialización y quedó casi inalterado (Merlan 2009: 69-70). Por último, aparte de los motivos geográficos y económicos, resulta imprescindible mencionar la vitalidad frente a la riqueza cultural y tradicional del mirandés. Este pequeño territorio vivió durante siglos relativamente aislado y conservó fiel e intensamente sus propias costumbres, su idioma y su estilo de vida rural. A pesar de la ausencia de una literatura escrita en mirandés hasta 1884, se consiguió transmitir oralmente de una generación a otra dichos componentes culturales. Por eso aún hoy en día las identidades de los mirandeses están marcadas por la historia y los rasgos culturales de su tierra natal (Merlan 2009: 442).

\subsection{Situación actual}

Mirando retrospectivamente hacia las últimas décadas, se nota claramente que la Ihéngua mirandesa, a diferencia del riodonorés y del guadramilés, ha recibido cada vez más atención debido a los siguientes eventos especiales: la introducción de enseñanza del mirandés como disciplina optativa (1986), el reconocimiento como lengua oficial de Portugal (1999) y la convención ortográfica del mirandés (1999).

Con respecto a la enseñanza, el mirandés ya existía en varios colegios trasmontanos como asignatura optativa más de diez años antes de la aceptación oficial como lengua minoritaria. No obstante, desde la normalización se enseña el mirandés no solo en algunos colegios de Miranda, sino «em todas as escolas do 
concelho de Miranda do Douro, da pré-primária ao $12^{\circ}$ ano, como disciplina de opcão» (Ferreira 2010).

Además, el reconocimiento oficial del mirandés por la República de Portugal, o sea la entrada en vigor de la Ley núm. 7/99, no solo reguló asuntos escolares de la Terra de Miranda, sino que también aclaró la falta de precisión sobre la denominación del mirandés. A lo largo de los siglos XIX y XX, se utilizaban varios términos en la dialectología para referirse al mirandés como co-dialecto del portugués (Leite de Vasconcelos, ${ }^{5}$ citado en Lindley Cintra 1983: 125-126), dialecto leonés (Menéndez Pidal 1906: 132) o falares 'hablas', entre otras cosas debido a su bajo prestigio social en relación al portugués (Merlan 2009: 54). Aunque el mirandés ya era una lengua antes del año 1999 por tener una estructura gramatical, un vocabulario, propios rasgos lingüísticos, etc., ${ }^{6}$ fue la ley, la que oficialmente introdujo la designación de lengua o lengua minoritaria y la que contribuyó a que en la actualidad goce de mayor vitalidad (Merlan 2009: 89-95).

Otro evento que también jugó un papel decisivo para el camino evolutivo del idioma mirandés fue la publicación de la Convenção Ortográfica da Língua Mirandesa sólo poco después de la entrada en vigor de la Ley núm. 7/99. Los principales objetivos de dicha convención eran establecer "critérios unitários, sistemáticos, claros e económicos para escrever e ler em mirandês» (Barros y Raposo 1999: 6). A pesar de que ya existen textos escritos en mirandés desde $1884,{ }^{7}$ se puede decir que el reconocimiento oficial y la convención contribuyeron al auge de publicaciones mirandesas, tanto impresas como en línea, a principios del siglo XXI. El escritor e investigador mirandés Amadeu José Ferreira estuvo implicado en la mayoría de estas publicaciones: traducciones de Astérix y Obélix, crónicas, el primer Dicionário de Mirandês-Português (DMP) disponible en la red, etc. En cuanto al boom de publicaciones en línea, hay que mencionar que la mayor parte de las páginas web o

\footnotetext{
${ }^{5}$ José Leite de Vasconcelos fue un filólogo e investigador lingüístico portugués que destacó la existencia del mirandés a fines del siglo XIX: «Não é o português a única língua usada em Portugal [...]; fala-se aquí também o mirandês» (Leite de Vasconcelos 1882: 7-8).

${ }^{6}$ Canal de História (2008).

${ }^{7}$ Por ejemplo, la antología poética de José Leite de Vasconcelos, publicada en 1884 y titulada Froles Mirandezas.
} 
bien ya no existe, o bien no es actualizada regularmente. ${ }^{8}$ Las páginas web, en cambio, que son modernizadas con frecuencia (por ejemplo la Biquipédia o el blog frolesmirandesas.blogspot.com) ofrecen una variedad de información tanto sobre la cultura, literatura y tradición mirandesa como sobre la historia (Ferreira 2010).

\section{Léxico patrimonial mirandés: análisis del corpus}

El siguiente apartado se dedica al análisis de palabras escogidas de un corpus representativo del mirandés contemporáneo, concretamente de la obra titulada $O$ Lodo e as Estrelas/L Lhodo i las Streilhas ( $\left.{ }^{4} 2016\right)$ de José Telmo Ferraz. Como hemos anotado en la introducción, se trata de una obra bilingüe, publicada en 2016, originalmente escrita en portugués en el año 1960. Consiste en una colección de poemas del padre José Telmo Ferraz que incluye una traducción mirandesa realizada por el escritor mirandés Fracisco Niebro, pseudónimo de Amadeu Ferreira. ${ }^{9}$

Resulta obvia la afirmación de que la lengua mirandesa guarda claras semejanzas con el portugués y el español. De hecho, un gran número de palabras mirandesas son idénticas o bien a las del español, ${ }^{10}$ o bien a las del portugués. ${ }^{11}$ Incluso hay casos en que los tres idiomas comparten la misma ortografía. ${ }^{12} \mathrm{Si}$ bien la palabra mirandesa es idéntica al español o portugués, en la mayoría de los casos las tres lenguas muestran similitudes, por lo menos con respecto a la representación escrita.

A continuación, tras esbozar la metodología de análisis aplicada (§3.1.), se analizarán las palabras de la lectura mirandesa y se harán algunos comentarios

\footnotetext{
${ }^{8}$ Algunas incluso desde hace más de diez años.

${ }^{9}$ Amadeu Ferreira ha sido considerado como uno de los especialistas del mirandés, destacando su labor como defensor y cuidador de la lengua, así como también la figura de escritor e investigador de la variedad mirandesa.

${ }^{10}$ Del análisis de $O$ Lodo e as Estrelas / L Lhodo i las Streilhas se extrae que forman parte del léxico mirandés palabras como, por ejemplo: palabra, tiempo, sangre, miedo, mano, arena, pan, etc.

${ }^{11}$ Palabras coincidentes entre mirandés y portugués son, por ejemplo: nome, a gralha, moço, abraçar, chaga, fumo, punho, etc.

12 Algunos de los términos idénticos recogidos en mirandés, portugués y español son: música, sabor, triste, mina, tacho, casa, etc.
} 
léxicos a partir de la consulta en varios diccionarios (§3.2.). El análisis compara los términos del mirandés sólo con el portugués y el español; por el momento, no se ha considerado el contraste con otras lenguas románicas, ya que estas dos lenguas muestran la mayor conexión lingüística con el mirandés. Para las correspondencias portuguesas se ha tomado la traducción que nos ofrece la lectura. Con respecto a los equivalentes españoles, se ha utilizado el Dicionário Online PONS Espanhol-Português (PONS).

\subsection{Metodología de análisis}

A partir de un análisis léxico dividido en cuatro aspectos (Azevedo Sampaio 2017: 23-41), a saber, a) palabras mirandesas con rasgos del portugués y/o del español, b) lusismos en mirandés, c) hispanismos en mirandés y d) regionalismos, se van a comentar los escasos ejemplos de este último grupo, esto es, el de las palabras regionales que solamente aparecen en el texto mirandés.

El procedimiento de análisis ha sido el siguiente: primero, se ha presentado el conjunto de las palabras mirandesas (Tabla 1: origen, mirandés, portugués, español) que no solo contiene los étimos latinos, sino que también incluye sus correspondientes traducciones al español y al portugués. ${ }^{13}$ Además, también se ha utilizado el Dicionário de mirandês-português (DMP) para encontrar por ejemplo la familia léxica de ciertas palabras, sinónimos, etc.; después, se ha comentado el probable origen de estas palabras. Debido a la inexistencia de un diccionario etimológico mirandés, se han consultado diccionarios etimológicos de las lenguas en su entorno (español y portugués) para observar si comparten la misma procedencia. Con respecto al portugués, se ha tenido como base el Dicionário etimológico da língua portuguesa (DELP) de Machado y en el caso del español se ha usado el Diccionario crítico etimológico de la lengua castellana (DELC) de Corominas.

Aparte de los diccionarios mencionados, también se han consultado los siguientes corpus lingüísticos: el Corpus del Nuevo Diccionario Histórico del Español

\footnotetext{
${ }^{13}$ Cabe mencionar que se han empleado tanto los equivalentes portugueses traducidos en la obra, como el diccionario PONS para buscar los equivalentes en español.
} 
(CDH) y el Corpus Lexicográfico do Português (DIClweb) para tener en cuenta la documentación antigua, así como el Corpus del Español del Siglo XXI (CORPES) y el Corpus do Português $(\mathrm{CdP})^{14}$ para los testimonios actuales. En estas bases de datos no aparece ninguno de los seis casos analizados, lo cual, nos ha permitido establecer la hipótesis de que se trata de regionalismos, es decir, de mirandesismos.

\subsection{Léxico patrimonial mirandés: mirandesismos}

Según el Diccionario de la Lengua Española (DLE), el adjetivo patrimonial en el ámbito lingüístico se refiere a: «Dicho de una palabra: Que, a diferencia de los cultismos, ha seguido en su evolución las leyes fonéticas propias del idioma». Tras la observación de las palabras mirandesas con las portuguesas y con las españolas, y, a partir del análisis etimológico e histórico contrastivo, pueden clasificarse como léxico patrimonial mirandés los casos que se analizan a continuación.

En la Tabla 1 se encuentran los seis vocablos, que corresponden a las palabras mirandesas ${ }^{15}$ que muestran divergencias respecto a las formas léxicas portuguesas y españolas. Se trata de los casos que pueden asignarse al grupo de los regionalismos. Bajo este término se entiende, según el Diccionario del español actual (DEA) de Seco, Andrés y Ramos (2005: 3868): “Palabra o rasgo idiomático propios de una región”. En un estudio de los años sesenta, de Moura Santos (1967: 129) destacó que existía «un vigoroso "regionalismo lingüístico"» en la Terra de Miranda. Ella afirmó que éste surgió del orgullo que el pueblo mirandés tenía y todavía tiene ante sus costumbres y su lenguaje. A propósito de la existencia de regionalismos en mirandés, se han analizado las siguientes palabras (aguemitar - bózio - bardeiro - quaijeque merujeiro - prainada) y se han apuntado algunas notas sobre su probable procedencia y composición:

\footnotetext{
${ }^{14}$ Este corpus consta de dos partes: la primera parte ofrece textos publicados entre 1300-1900, mientras que la segunda presenta documentos a partir del siglo XX.

${ }^{15}$ En mirandés, como es propio de las variedades fronterizas, frecuentemente se utilizan términos similares a palabras de los idiomas en su entorno, aunque a veces modifican ciertas palabras con sus propios rasgos peculiares (p. ej. lat. vivĕre > mir. bibéncia; port. vivência; esp. vivencia).
} 


\begin{tabular}{|c|c|c|c|}
\hline latín & mirandés & portugués & español \\
\hline vomĭtāre & aguemitar & vomitar [vumi'tar] & vomitar \\
vōx, vōcis & bózio & grito ['gritu] & grito \\
verrĕre & bardeiro & vassoura [ve'sore] & escoba \\
quăš̆ & quaijeque & quase ['kwazə] & casi \\
plŭvĭa & merujeiro & chuva miudinha ['Juve] & Ilovizna \\
planu & prainada & planície [ple'nisjə] & planicie \\
\hline
\end{tabular}

Tabla 1. Origen, mirandés, portugués, español.

Dado que no existe ningún diccionario etimológico $-y$ mucho menos uno histórico del mirandés-, y teniendo en cuenta que la situación lexicográfica del mirandés tampoco goza de sólidos fundamentos ni de recopilación ni de descripción del léxico, en algunos casos, como en aguemitar, se han bosquejado algunas propuestas a partir de la observación y de la crítica contrastiva entre la forma en mirandés y sus equivalentes en portugués y en español.

\subsection{1 aguemitar (mir.): vomitar (port.) - vomitar (esp.)}

El vocablo mirandés aguemitar destaca por haber evolucionado de manera diferente en los otros idiomas: vomitar. Mientras que vomitar fue transmitido al castellano y portugués por vía culta, aguemitar presenta una «estropiação de palavras cultas» (Braga 2015).

Vemos, a continuación, el primero de los ejemplos obtenido del corpus. En la primera columna aparece la palabra mirandesa, mientras que en la segunda columna se observa la frase en portugués (Traducción al español: «El Zeca vomitó sangre. Una sangre viva»):

\begin{tabular}{|l|l|}
\hline \multicolumn{1}{|c|}{ Palabra mirandesa (Telmo, $\left.{ }^{4} 2016: 17\right)$} & Palabra portuguesa (Telmo, $\left.{ }^{4} 2016: 16\right)$ \\
\hline $\begin{array}{l}\text { L Zeca aguemitou sangre. Un sangre } \\
\text { bibo. }\end{array}$ & $\begin{array}{l}\text { O Zeca vomitou sangue. Um sangue } \\
\text { vivo. }\end{array}$ \\
\hline
\end{tabular}

Tabla 2. aguemitou (mirandés) - vomitou (portugués)

Aunque aguemitar también ha conservado la conjugación latina -are, se aprecian marcadas diferencias respecto a la raíz y a las dos primeras sílabas de la palabra: a-gue-mi-tar. Si la palabra hubiera seguido el mismo camino evolutivo que el 
portugués y el español, quizás habría surgido la palabra *bomitar u otra forma similar. A causa de que no haya ninguna otra lengua románica que tenga la misma grafía que el mirandés, se ofrecen tres hipótesis que pueden servir para entender la constitución de dicha palabra. Hay que tener en cuenta que, frecuentemente, en mirandés se tiende a conservar vocablos antiguos del portugués o del español ( $p$. ej. suidade, tembrar, sumana, etc.). Dicho esto, aguemitar no se recoge en ninguno de los corpus ni diccionarios consultados, aunque su presencia, en la lengua mirandesa, puede haber sido ejercida por la variante gomitar, que tenía un uso corriente durante los siglos XVI y XVII en España y también en Portugal. La forma con $\langle g->$ incluso sobrevivió «como vulgarismo usual en Aragón, Santander y muchas partes de España» (DELC: 761). Suponiendo que la palabra realmente se hubiera constituido por influjo de gomitar, quedaría por justificar la prótesis de la $a$-.

Una segunda posibilidad puede ser que el término mirandés se formara por la influencia de palabras semejantes que entran en el contexto de vomitar: angustia, angustiar, agonía, agoniar 'sentir náusea o mareo' (término port.), etc. Esto explicaría por lo menos la existencia de la consonante $\langle\mathrm{g}\rangle$, así como el desarrollo de la $a$ - inicial.

Por último, un tercer supuesto puede encontrarse en la relación que se establece entre vómito con la palabra agua, entendidos elementos líquidos. Las primeras dos sílabas de aguemitar reflejan nítidamente una asociación con la palabra agua.

\subsection{2 bózio (mir.): grito (port.) - grito (esp.)}

La segunda palabra de la Tabla 1 es el sustantivo mirandés bózio. Su correspondencia en portugués y español es grito. Tanto en el caso anterior, como en éste, coinciden los equivalentes en ambas lenguas.

Esta palabra aparece en el siguiente contexto (traducción al español: «Vamos callados. Este silencio despertará a los que duermen porque es el grito acusador, que no es violento, pero que clama por justicia»: 


\begin{tabular}{|l|l|}
\hline Palabra mirandesa (Telmo ${ }^{4}$ 2016: 125) & Palabra portuguesa (Telmo ${ }^{4} 2016:$ 124) \\
\hline Bamos calhados. Este silenço spertará & Vamos em silêncio. Este silêncio \\
Is que drúmen porque ye I bózio & acordará os que doermem porque é um \\
acusador, que nun ye biolento, mas & grito acusador, que não é violento, mas \\
bózia por justícia. & clama por justiça. \\
\hline
\end{tabular}

Tabla 3. bózio (mirandés) - grito (portugués)

A primera vista parece que las palabras no estén relacionadas entre sí, pero al consultar el DELC de Corominas, se advierte claramente que existe una cierta conexión lingüística: el término grito derivó del verbo gritar y éste proviene del latín quiritāre 'dar grandes voces'. La raíz de bózio parece ser el sustantivo boç- 'voz' y este también aparece en la definición de gritar. Según Corominas (DELC: 791-792), el castellano y el portugués se individualizan por poseer el término grito, el cual aparece menos frecuentemente en los restantes idiomas romances.

En el caso del vocablo mirandés bózio (derivado del verbo boziar) se observa que no surgió del latín quiritāre, sino del latín vōx, vōcis (Leite de Vasconcelos 1901: 172). Por lo tanto, la lengua mirandesa se distancia del portugués y del español por tener étimos distintos respecto a esta palabra. En suma, se puede decir que en mirandés se produjo el término bózio, ${ }^{16}$ así como boziar 'gritar, vocear' y bozeiron 'vozarrón', a partir de la raíz sustantiva boç-17 'voz-', mientras que el portugués y el español no construyeron el sustantivo equivalente a bózio a partir de esta raíz, sino que prefirieron, como ha sido señalado anteriormente, conservar grito.

\subsection{3 bardeiro (mir.): vassoura (port.) - escoba (esp.)}

Este sustantivo (bardeiro) equivale al portugués vassoura y al español escoba. En este tercer caso, no coinciden tampoco las formas portuguesa ni española.

El ejemplo, lo encontramos en el siguiente contexto (traducción al español: «Ella puso, en una esquina, la escoba»:

\footnotetext{
${ }^{16}$ Se trata del fenómeno ortográfico del mirandés por el cual se sustituye la letra $\langle\mathrm{v}\rangle$ por la letra $\langle\mathrm{b}\rangle$ en todas las palabras de origen latino.

17 Durante la Baja Edad Media existían las dos grafías boz y voz en España (DELC: 764).
} 


\begin{tabular}{|l|l|}
\hline \multicolumn{1}{|c|}{ Palabra mirandesa (Telmo ${ }^{4}$ 2016: 49) } & Palabra portuguesa (Telmo ${ }^{4} 2016: 48$ ) \\
\hline $\begin{array}{l}\text { Eilha pousou, a ua squina, I bardeiro de } \\
\text { scobas. }\end{array}$ & $\begin{array}{l}\text { Ela poisou, a um canto, a vassoura de } \\
\text { giesta. }\end{array}$ \\
\hline
\end{tabular}

Tabla 4. bardeiro (mirandés) - vassoura (portugués)

Debido al hecho de que, a primera vista, no se verifique ninguna relación entre los tres equivalentes, se analiza el término mirandés con respecto a su raíz y su probable formación. En primer lugar, es necesario separarlo en sílabas: bar-dei-ro. La primera sílaba bar- se puede asociar con el verbo barrer (port. varrer; esp. barrer) que proviene del latín verrĕre y cuyo significado equivale al del portugués y del español. Mientras que en portugués sólo hubo un cambio vocálico de verr- en varr-, en mirandés y en español se observa además un cambio fonético (verr- > barr-) (DELC: 411).

De acuerdo con que bardeiro se formara a partir de la raíz verbal barrer, se puede suponer que los mirandeses crearan una nueva palabra que definiera el objeto que se necesita para barrer, añadiendo el sufijo -eiro ${ }^{18}$ a la raíz. Aunque no es evidente a primera vista, el término portugués vassoura también se formó de manera semejante al mirandés, es decir, a partir del mismo étimo: vassoura < *versōrĭa < lat. verrĕre (DELP: 378). Escoba, por el contrario, surgió de un étimo distinto (lat. scōpa 'arbusto, conjunto de ramos de planta') y se desarrolló durante los últimos siglos hasta alcanzar su significado actual (DELC: 350$)$.

Por último, hay que mencionar que entre la familia de palabras de barrer o varrer existen algunos sustantivos, tanto en portugués como en español, que se aproximan a la grafía de bardeiro, pero no a su sentido: barredero, barrendero (port. varredor).

\footnotetext{
${ }^{18}$ Según Gómez Bautista (2013: 203-205), este sufijo generalmente se encuentra en nombres de plantas y árboles, designaciones de algunas profesiones y en nombres gentilicios.
} 
3.2.4 quaijeque (mir.): quase (port.) - casi (esp.)

Otro caso es el adverbio mirandés quaijeque que según Gómez Bautista (2013: 273-274), puede ser denominado como «composición ortográfica» por consistir en la unión de un adverbio (quaije) y de una conjunción (que). El portugués y el español presentan los semicultismos quase y casi que proceden del latín quăsi 'como si' (DELC: 718).

El contexto en el que aparece la palabra es el siguiente (traducción al español: «Apuntes simples, en lo cotidiano, de cosas tan simples, casi banales - pero apuntes nuestros, reales.»):

\begin{tabular}{|l|l|}
\hline \multicolumn{1}{|c|}{ Palabra mirandesa (Telmo ${ }^{4}$ 2016: 15$)$} & \multicolumn{1}{c|}{ Palabra portuguesa (Telmo ${ }^{4}$ 2016: 14) } \\
\hline $\begin{array}{l}\text { Apuntamientos simpres, to ls dies, de } \\
\text { cousas tan simpres, quaijeque banales - } \\
\text { mas apuntamientos nuossos, sérios. }\end{array}$ & $\begin{array}{l}\text { Apontamentos simples, no quotidiano, } \\
\text { de factos tão simples, quase banais - } \\
\text { mas nossos, reais. }\end{array}$ \\
\hline
\end{tabular}

Tabla 5. quaijeque (mirandés) - quase (portugués)

Se observa la existencia de una variante gráfica de este término mirandés porque la lectura no solo ofrece quaijeque (por ejemplo, Telmo ${ }^{4} 2016$ : 17), sino también quaije (por ejemplo, Telmo ${ }^{4} 2016: 45$ ) y en ambos casos es traducido por quase 'casi'. El vocablo podría ser un regionalismo, ya que no se encuentra en otra lengua y tampoco en las variantes antiguas del portugués y del español: cuasi, quassi, casy, caise, caisi (DELC: 718; DELP: 11).

Quizá la existencia de la forma aglutinada quaijeque al lado de quaije se deba al elemento de composición culta quase- 'como si, dado que' que sobrevivió en algunos contextos del portugués, como, por ejemplo: quase-contrabando, quase-contrato (DELP: 11). Debido a la carencia de un diccionario etimológico del mirandés, el origen y la composición de esta palabra sigue siendo un caso no resuelto. 


\subsection{5 merujeiro (mir.): chuva miudinha (port.) - llovizna (esp.)}

En cuanto al próximo término merujeiro, cabe destacar que tanto el texto como la consulta en el DMP ofrecen una traducción al portugués que está compuesta de dos palabras: chuva miudinha. En español, llovizna parece ser el término más adecuado para expresar el sentido de 'lluvia menuda que cae blandamente' (DLE).

La palabra aparece en el siguiente contexto (traducción al español: «Noche sin estrellas. Una llovizna. Las luces del barrio relucían»):

\begin{tabular}{|l|l|}
\hline Palabra mirandesa (Telmo ${ }^{4}$ 2016: 125) & Palabra portuguesa (Telmo $\left.{ }^{4} 2016: 124\right)$ \\
\hline $\begin{array}{l}\text { Nuite sien streilhas. Un merujeiro. Las } \\
\text { Ihuzes de I bárrio relhuzien. }\end{array}$ & $\begin{array}{l}\text { Noite sem estrelas. Uma chuva } \\
\text { miudinha. As luzes do bairro cintilavam. }\end{array}$ \\
\hline
\end{tabular}

Tabla 6. merujeiro (mirandés) - chuva miudinha (portugués)

A causa de que las etimologías de las palabras Iluvia y chuva (< lat. plŭvĭa) no sirven para poder explicar la formación de merujeiro, cabe considerar la búsqueda de sinónimos y términos que podrían estar relacionados con este vocablo. A propósito del portugués, el sustantivo chuveiro 'ducha', por ejemplo, puede guardar cierta conexión lingüística con merujeiro, puesto que la salida del agua al ducharse puede compararse a la acción de 'proyección de agua'19 (DLE). Esta palabra portuguesa por lo menos permite una aproximación a la presencia del sufijo -eiro.

Con respecto a la parte inicial de la palabra (meru-), se coteja una gran semejanza ortográfica con el adjetivo mero (esp. y port.). Ahora cabe preguntarse si el vocablo merujeiro puede haber surgido a partir de este adjetivo, por ejemplo, en frases como la mera chuva 'la mera lluvia', y por influjo de chuveiro, alcanzando su grafía y valor semántico actuales.

\footnotetext{
${ }^{19}$ En algunas variedades del español, también la palabra lluvia puede ser equivalente de ducha, como se indica en la acepción número 4 del DLE para el español de Argentina y de Uruguay (DLE).
} 
3.2.6 prainada (mir.): planície (port.) - planicie (esp.)

Por último, se analiza concisamente el término mirandés prainada que no se ha encontrado en ninguna otra lengua iberorrománica. El portugués y el español comparten la misma palabra con casi la misma grafía (port. planície; esp. planicie).

En el corpus, la palabra aparece en el siguiente contexto (traducción al español: «En aquella hora, allá lejos, las senaras extendían las mantas en la planicie, para dormir la noche de un solo sueño.»:

\begin{tabular}{|l|l|}
\hline \multicolumn{1}{|c|}{ Palabra mirandesa (Telmo ${ }^{4}$ 2016: 49) } & Palabra portuguesa (Telmo ${ }^{4}$ 2016: 48) \\
\hline $\begin{array}{l}\text { Naqueilha hora, alhá loinge, las senaras } \\
\text { stendien las mantas na prainada, para } \\
\text { drumíren la nuite toda dun suonho. }\end{array}$ & $\begin{array}{l}\text { Naquela hora, lá longe, as searas } \\
\text { estendiam as mantas na planície, para } \\
\text { dormirem a noite dum só sono. }\end{array}$ \\
\hline
\end{tabular}

Tabla 7. prainada (mirandés) - planície (portugués)

Es posible que la formación de prainada se explique por influjo de términos sinonímicos del portugués y del español, es decir, palabras que encajen con el significado 'terreno llano, especialmente de gran extensión' (DLE): esp. llanada; port. praino.

Sin duda alguna, existen otros sinónimos de planicie; sin embargo, las grafías de estos dos vocablos parecen ser útiles para especular sobre la probable formación y procedencia de prainada. El primer sinónimo llanada, que antiguamente tenía el duplicado planada (DELC: 156), puede explicar el sufijo -ada que según Gómez Bautista (2013: 192) es «muy productivo en mirandés». En cuanto al término portugués praino, que también forma parte del léxico mirandés en el sentido de 'altiplano', cabe mencionar que se trata de una forma alterada de plaino, término epentético de plano < lat. plānus, según nos indica el Novo dicionário da língua portuguesa (NDLP: 1097; 1125) de Buarque de Holanda Ferreira.

Aunque la mayoría de las palabras portuguesas del grupo inicial latino plmuestran un desarrollo hacia el grupo ch-, hay casos en los cuales se mantuvo pl- y otros que evolucionaron a pr-. Según este último grupo, considerado como propia característica del gallegoportugués, resulta obvia la conservación de dicha 
característica en algunos vocablos modernos del portugués e incluso del mirandés (Teyssier ${ }^{2} 1984:$ 14-15). Por lo tanto, debido al hecho de que el mirandés también posee la palabra praino, es muy posible que ésta fuera importada por la influencia de los portugueses.

Con respecto al sufijo -ada, parece apropiado barajar la posibilidad del influjo del término español llanada (duplicado antiguo planada). Por otro lado, quizás los mirandeses simplemente añadieran -ada a la raíz sustantiva praino, ya que este sufijo aparece con alta frecuencia en su idioma.

\section{Conclusión}

El objetivo de este estudio ha sido exponer y observar las palabras regionales recopiladas de un texto literario en mirandés, producto de una traducción original portuguesa y que nos ha llevado a una investigación de carácter contrastivo. Este análisis nos ha permitido comprobar que se muestran preferencias opuestas a los equivalentes de sus lenguas en contacto (portugués y español).

Los seis vocablos analizados pueden clasificarse como regionalismos mirandeses dado que no existen en ninguna otra variedad, por lo menos en cuanto a su forma escrita; por tanto, la morfología de estas palabras expresa e indica cierta peculiaridad, en este caso, del léxico patrimonial mirandés.

Al igual que puede hablarse de lusismos e hispanismos mirandeses, cabe preguntarse si se pueden designar estas palabras como mirandesismos. Aunque este término no lo hemos encontrado definido en ningún diccionario, fue utilizado por Leite de Vasconcelos (1901: 21 y 305) en la obra Estudos de philologia mirandesa. El autor utiliza este término para referirse a palabras cuyas formaciones no están claras o que indican un cambio evolutivo distinto al español y/o al portugués. Debido a la presencia de vocablos propios del mirandés, podría justificarse la denominación de mirandesismos, al igual que se aplican también los conceptos de lusismos e hispanismos, en casos similares, para hablar de términos peculiares de Portugal y 
España. Por otro lado, somos conscientes de que no hay que confundir esta nomenclatura lingüística con otras posibilidades que se ofrecen a partir de la búsqueda en internet: el término mirandesismo también se encuentra, en el ámbito del deporte, para referirse a todos los seguidores del equipo de fútbol de Miranda de Ebro, otra ciudad española, que se encuentra en la provincia de Burgos y que comparte el mismo topónimo.

Como se ha señalado a lo largo de este estudio, la lengua mirandesa carecía de una literatura escrita hasta finales del siglo XIX, por lo que tampoco había recibido atenciones por parte de los lingüistas. A pesar de una creciente vitalidad literaria, cultural y administrativa en el siglo siguiente, en el cual diversos investigadores se ocuparon del origen y de las similitudes del mirandés respecto a las lenguas con las que ha entrado en contacto, los regionalismos mirandeses han sido y siguen siendo elementos desatendidos en la lingüística. Debido a esto, la presente investigación ha pretendido señalar esta carencia de estudios y dedicar estas líneas al análisis de esta parcela del léxico mirandés, tomando como corpus una obra literaria actual.

\section{Referencias bibliográficas}

Azevedo MalA, Clarinda de (1996) «Mirandés», in Manuel Alvar (dir.), Manual de dialectología hispánica. El español de España, Barcelona: Ariel, 159-170.

AzeVedo SAMPAIO, Melanie (2017) L mirandés. El desarrollo de una variedad lingüística en la frontera hispano-portuguesa, Trabajo Fin de Grado (TFG - Bachelor of Education), Universidad de Paderborn.

BarRos FerreIRA, Manuela y Domingos Raposo (coords.) (1999) Convenção Ortográfica da Língua Mirandesa, Miranda do Douro/Lisboa: Câmara Municipal de Miranda do Douro/Centro de Linguística da Universidade de Lisboa.

BECKER, Ídel (1983) Grande dicionário latino-americano português-espanhol, Sao Paulo: Nobel [GDLP].

BRAGA, Franklim (2015) "Sobre "O Falar de Riba Côa" - Letra A». Capeia Arraiana, [http://capeiaarraiana.pt/2015/04/10/sobre-o-falar-de-riba-coa-letra-a/] (Consulta: 23/02/2018). 
BuARQUe de Holanda FerreirA, Aurélio (1985) Novo dicionário da língua portuguesa, Rio de Janeiro: Nova Fronteira [NDLP].

CANAL DE HISTÓRIA (2008) «Mirandês outra língua (1de7)». Youtube, 2:26-2:42, [https://www.youtube.com/watch?v=3SIv2sF5jOM] (Consulta 23/02/2018).

CENTRO DE LINGUÍSTICA DA UNIVERSIDADE DE LISBOA / UNIVERSIDADE DE AVEIRO: Corpus Lexicográfico do Português, [http://clp.dlc.ua.pt/Inicio.aspx] (Consulta 23/02/2018) [DIClweb].

COROMINAS, Joan (1976) Diccionario crítico etimológico de la lengua castellana, Madrid: Gredos [DELC].

Davies, Mark y Michael J. FerreiRA (2006-) O Corpus do Português: 45 milhões de palavras, 1300s-1900s, [http://www.corpusdoportugues.org/xp.asp] (Consulta 23/02/2018) $[\mathrm{CdP}]$.

Dicionário Online PONS Espanhol-Português, [http://pt.pons.com/tradu\% C3\%A7\%C3\%A3o/portugu\%C3\%AAs-espanhol] (Consulta 23/02/2018) [PONS].

Ferreira, Amadeu y José Pedro Cardona Ferreira (2007) Dicionário de Mirandês-Português, [http://www.mirandadodouro.com/dicionario/] (Consulta 23/02/2018) [DMP].

FERREIRA, Amadeu (2010) "O mínimo sobre a língua mirandesa», Revista do Festival Intercéltico, [http://studosmirandeses.blogs.sapo.pt/1294.html] (Consulta 23/02/2018).

GÓMEZ BAUTISTA, Alberto (2013) El mirandés: contexto y procesos de formación de palabras, Tesis doctoral, Universidad Complutense de Madrid.

INSTITUTO DE INVESTIGACIÓN RAFAEL LAPESA DE LA REAL ACADEMIA ESPAÑOLA (2013) Corpus del nuevo diccionario histórico (CDH), [http://web.frl.es/CNDHE/view/inicioExterno.view] (Consulta 23/02/2018) [CDH].

Leite de VAsconcelos, José (1882) O dialecto mirandês. Contribução para o estudo da dialectologia românica no domínio glotológico hispano-lusitano, Porto: Livraria Portuense.

Leite de Vasconcelos, José (1901) Estudos de Philologia Mirandesa, vol. II, Lisboa: Imprensa Nacional.

LINDLEY CINTRA, Luís Filipe (1983) Estudos de dialectologia portuguesa, Lisboa: Sá da Costa.

MACHADO, José Pedro (1977) Dicionário etimológico da língua portuguesa: com a mais antiga documentação escrita e conhecida de muitos dos vocábulos estudados, Lisboa: Livros Horizonte [DELP].

MEDINA LóPEZ, Javier (2002) Lenguas en contacto, Madrid: Arco/Libros. 
MENÉNDEZ PIDAL, Ramón (1906) «El dialecto leonés», Revista de archivos, bibliotecas y museos, XIV, 128-172.

MERLAN, Aurelia (2009) El mirandés: situación sociolingüística de una lengua minoritaria en la zona fronteriza portugués-española, Uviéu: Academia de la Llingua Asturiana.

MoURA SANTOS, María José de (1967) Os falares fronteiriços de Trás-os-Montes, Coimbra: Instituto de Estudos Românicos.

Real Academia Española: Banco de datos (CORPES XXI). Corpus del Español del Siglo XXI, [http://web.frl.es/CORPES/org/publico/pages/cita/cita.view] (Consulta 23/02/2018) [CORPES].

REAL ACADEMIA ESPAÑola ( $\left.{ }^{23} 2014\right)$ Diccionario de la lengua española, Madrid: Espasa [DLE].

SECO, Manuel, ANDRÉs, Olimpia y Gabino RAMOs (2005[1999]) Diccionario del español actual, Madrid: Aguilar [DEA].

Telmo FerRAZ, José ( $\left.{ }^{4} 2016[1960]\right)$ O Lodo e as Estrelas / L Lhodo i las Streilhas, traducción al mirandés de Fracisco Niebro, Lisboa: Âncora.

TEYSSIER, Paul (21984) História da língua portuguesa, Lisboa: Sá da Costa. 